EPJ Web of Conferences 78, 04003 (2014)

DOI: $10.1051 /$ epjconf/20147804003

(C) Owned by the authors, published by EDP Sciences, 2014

\title{
The role of the Wigner function in charged-particle beam trans- port
}

\author{
Renato Fedele ${ }^{1,2, a}$, Margarita A. Man'ko ${ }^{3}$, Vladimir I. Man'ko ${ }^{3}$, and Sergio De Nicola ${ }^{4,2}$ \\ ${ }^{1}$ Dipartimento di Fisica, Universitá di Napoli Federico II, Napoli Italy \\ ${ }^{2}$ INFN Sezione di Napoli, Napoli, Italy \\ ${ }^{3}$ P.N. Lebedev Physical Institute, Moscow, Russia \\ ${ }^{4}$ SPIN-CNR, Napoli, Italy
}

\begin{abstract}
The role of the Wigner function in the dynamics of charged particle beams in high-energy accelerating machines is discussed. This is done within the quantum-like description of the thermal wave model (TWM). A brief review of the numerical experiments showing satisfactory agreement between TWM and the particle tracking simulations is presented. A simple analysis in phase space in terms of the Wigner quasidistribution, showing that TWM is capable of reproducing the beam dynamics in the presence of the space charge effects, is put froward.
\end{abstract}

The branch of electron optics describing the transport of non-laminar paraxial high-energy charged-particle beams involves the dynamics of an extremely large number of charged particles that is mainly dominated by the electromagnetic interactions, while the effects of the temperature cannot be neglected. The behavior of such a system is collective and affected by the thermal spreading among the particles (thermal regime). This discipline has been strongly developed in particle accelerator physics [1].

A peculiar aspect of the electron optics of non-laminar beams is the mixing of the particle trajectories, i.e., mixing among the electron rays [1]. In paraxial beams, this effect causes a slight random deviation of the electron rays slopes (with respect to the propagation direction). In vacuo, the electron ray slopes are Boltzmann-distributed, hence the instantaneous transverse momentum spread (normalized to the longitudinal relativistic particle momentum) is $\sigma_{p} \sim v_{T} / c \ll 1\left(v_{T}\right.$ and $c$ being the transverse thermal velocity and the speed of light, respectively). However, it is a matter of fact, both theoretically predicted and experimentally observed, that during the beam motion the instantaneous spread of the electron rays positions in the transverse plane, say $\sigma$, is related to $\sigma_{p}$ by the following inequality:

$$
\sigma \sigma_{p} \geq \epsilon
$$

where $\epsilon$ is the transverse beam emittance, which measures the conserved area of the phase space region accessible to the system.

Consequently, the thermal spreading introduces an uncertainty relation between electron ray positions and ray slopes. Therefore, the envelope of all electron rays (paraxial electron ray pattern) appears fully similar to the one generated by the ray envelope in the paraxial light beam (here the random mixing of the light rays is caused by the paraxial diffraction). This analogy, that is fully coherent

ae-mail: renato.fedele@na.infn.it 
with the isomorphic correspondence between optics and mechanics, has been stressed to point out that the statistical behavior of the charged particles in a paraxial beam emulates the paraxial diffraction of the e.m. radiation. Then, we may refer the paraxial electron ray pattern to as paraxial diffraction-like electron ray pattern.

Since inequality (1) is the analog of the quantum uncertainty relation, a quantum-like model for charged-particle beam transport has been earlier formulated in terms of a complex function, called the beam wave function (BWF), whose squared modulus is proportional to the beam density [2]. The model is known as Thermal Wave Model (TWM) and the spatiotemporal evolution of the BWF is governed by the following 2D Schrödinger-like equation [2]

$$
i \epsilon \frac{\partial \Psi}{\partial z}=-\frac{\epsilon^{2}}{2} \nabla_{\perp}^{2} \Psi+U\left(\mathbf{r}_{\perp}, z\right) \Psi
$$

where the transverse emittance $\epsilon$ replaces $\hbar, \nabla_{\perp}$ is the transverse component of the gradient operator, $\mathbf{r}_{\perp}=(x, y)$ is the transverse component of the position vector, the longitudinal coordinate $z$ plays the role of the time-like variable and the single-particle effective potential energy $U\left(\mathbf{r}_{\perp}, z\right)$, in principle, accounts for both external and self-consistent interactions. When the self-consistent interactions are present, then Eq. (2) has to be coupled with the related potential energy.

With the use of the Wigner transform, i.e.,

$$
W\left(\mathbf{r}_{\perp}, \mathbf{p}_{\perp}, z\right)=\frac{1}{(2 \pi \epsilon)^{2}} \int \Psi^{*}\left(\mathbf{r}_{\perp}+\frac{\mathbf{y}}{2}, z\right) \Psi\left(\mathbf{r}_{\perp}-\frac{\mathbf{y}}{2}, z\right) \exp \left(i \frac{\mathbf{p}_{\perp} \cdot \mathbf{y}}{\epsilon}\right) d^{2} y,
$$

the quantum-like description provided by TWM has also been extended to $\left(\mathbf{r}_{\perp}, \mathbf{p}_{\perp}\right)$ phase space $\left[\mathbf{p}_{\perp}=\right.$ $(d x / d z, d y / d z)$ being the transverse single-particle momentum, conjugate of $\left.\mathbf{r}_{\perp}\right]$. Then, the Wigner quasidistribution $W$ is governed by the following von Neumann-like equation:

$$
\frac{\partial W}{\partial z}+\mathbf{p}_{\perp} \cdot \nabla_{\perp} W+\frac{i}{\epsilon}\left[U\left(\mathbf{r}_{\perp}+(i \epsilon / 2) \nabla_{p_{\perp}}\right)-U\left(\mathbf{r}_{\perp}-(i \epsilon / 2) \nabla_{p_{\perp}}\right)\right] W=0 .
$$

Provided that $W$ is normalized to 1, i.e., $\int W\left(\mathbf{r}_{\perp}, \mathbf{p}_{\perp}, z\right) d^{2} r_{\perp} d^{2} p_{\perp}=1$, the quantities $P_{r_{\perp}}\left(\mathbf{r}_{\perp}, z\right)=$ $\int W\left(\mathbf{r}_{\perp}, \mathbf{p}_{\perp}, z\right) d^{2} p_{\perp}$ and $P_{p_{\perp}}\left(\mathbf{r}_{\perp}, z\right)=\int W\left(\mathbf{r}_{\perp}, \mathbf{p}_{\perp}, z\right) d^{2} r_{\perp}$ represent, for any $z$, the probability to find an electron ray at the position $\mathbf{r}_{\perp}$ in the transverse plane and the probability to find an electron ray with a slope $d \mathbf{r}_{\perp} / d z=\mathbf{p}_{\perp}$, respectively. Note that, the meaning of $W\left(\mathbf{r}_{\perp}, \mathbf{p}_{\perp}, z\right)$ does not correspond to a classical positive definite probability as in the case of the Boltzmann distribution. According to Eq. (1), $W$ takes suitably into account the uncertainty between positions and slopes of the electron rays. Therefore the loss of information within cells of the phase space with extent of the order of $\epsilon$ justifies the possibility that $W$ assumes negative values.

With the use of TWM, a number of linear and nonlinear problems in conventional and plasmabased particle acceleration were successfully described [3-10].

Several comparisons between TWM and particle tracking code simulations have been carried out for different problems of beam transport in the presence of quadrupole-like devices with small multipolar aberrations (sextupole and octupole deviations), i.e., by taking a $(1+1) \mathrm{D}$ external potential of the form

$$
U(x, z)=\frac{1}{2 !} k_{1} x^{2}+\frac{1}{3 !} k_{3} x^{3}+\frac{1}{4 !} x^{4} .
$$

The predictions of TWM were the output distributions, in configuration space or in phase space, of an initially given Gaussian beam, after passing the entire arrangements of devices, ended usually with a subsequent drift space. These predictions have been compared to the ones of a standard kick code, 
showing a remarkable agreement [7]. Such an analysis has been extended to the phase space by means of the Wigner quasidistribution with more flexibility with respect to the sequence of the multipolar devices and their strengths. Additionally, the analytical predictions have also been extended to the Husimi function [11]. The outputs of both Wigner and Husimi functions have been compared to the tracking of 700,000 particles [9]. An excellent agreement for all the numerical experiments (involving different optical devices arrangements) has been observed in phase space for the contour $1 \sigma$ and $2 \sigma$, even when the sextupole and octupole strengths were not so small to justify a first order effects. At $3 \sigma$, some discrepancies, more pronounced in the case of the Wigner function, have been observed; however, they appear in the periphery of the distribution where regions with negative phase-space density yield an unrealistic distortion of the phase-space countour. By the use of the Husimi function, instead, this effect is largely smoothed out [9]. More recent comparisons between TWM predictions and the particle tracking PARMILA carried out in configuration space, have been shown further remakable agreements $[12,13]$. This has been accomplished by using a functional method, that describes the beam optical elements separately with a kernel for a component, to find the predictions of TWM in the presence of quadrupole magnets beyond the thin lens approximation and a drift space [12]. The comparison has lead to conclude that TWM can successfully describe the PARMILA simulation results through a focusing/defocusing (FODO) lattice structure for the Gaussian input beam without space charge effects [12]. Furthermore, the same authors ([13]) have extended their comparison including sextupole and octupole perturbations, leading to conclude that TWM successfully explains the PARMILA simulation results for proton beams without space charge effects in a lattice with 10 FODO cells [13].

We observe that all the comparisons described above do not account for the space charge effects. Then, it is natural to implement those investigations with the inclusions of the space charge effects. The investigation should be carried out in both configuration and phase space, and this is planned for future works. For the time being, an analytical approach seems to be possible by considering the propagation a relativistic charged-particle beam in vacuo in the presence of a strong uniform magnetic field, oriented along the beam propagation direction, i.e., $\mathbf{B}_{0}=B_{0} \hat{z}$. In addition, we assume that the space charge effects (i.e., the total Lorentz force on a single beam particle accounts for the collective electric and magnetic field created by both charge and current distributions within the beam) are not negligible. This way, it is easy to see that, in cylindrical symmetry, the effective single-particle potential energy in Eqs. (2) and (4) is given by

$$
U\left(r_{\perp}, z\right)=\frac{1}{2} K r_{\perp}^{2}+U_{s c}\left(r_{\perp}, z\right)
$$

where $K=\left(e B_{0} / 2 m_{0} \gamma_{0} c^{2}\right)^{2}$ and the space charge potential energy, i.e., $U_{s c}$, satisfies the following Poisson's equation, viz.,

$$
\frac{1}{r_{\perp}} \frac{\partial}{\partial r_{\perp}}\left(r_{\perp} \frac{\partial U_{s c}}{\partial r_{\perp}}\right)=\lambda \int W\left(\mathbf{r}_{\perp}, \mathbf{p}_{\perp}, z\right) d^{2} p_{\perp}
$$

with $\lambda=-2 \pi q^{2} N / \sigma_{z} m_{0} \gamma_{0}^{3} c^{2}$. Here, $N$ is the number of the beam particles, $\sigma_{z}$ is the beam length, $q$ and $m_{0}$ are, respectively, charge and rest mass of the single particle and $\gamma_{0}$ is the relativistic gamma factor. We want to show that the Wigner-Poisson pair of equations (4) and (7) is, at least, capable of describing analytically the motion of a beam, that is initially in the ground state, in the presence of the space charge. We show that the result does not differ from the one given by the conventional description. This is done taking the aberration-less approximation, i.e., for a stable condition of a strongly localized beam around $r_{\perp}=0$, the total potential energy $U\left(r_{\perp}, z\right)$ has a minimum in $r_{\perp}=0$ and can be suitably expanded up to the second power in $r_{\perp}$. When this is self-consistently done, we 
obtain:

$$
U\left(r_{\perp}, z\right) \approx \frac{1}{2} \bar{K}(z) r_{\perp}^{2}
$$

where, to ensure that Wigner-Poisson system admits bounded states, the condition $\bar{K}>0$ must be imposed, and Eq. (4) is reduced to:

$$
\frac{\partial W}{\partial z}+\mathbf{p}_{\perp} \cdot \nabla_{\perp} W-\bar{K}(z) \mathbf{r}_{\perp} \cdot \nabla_{p_{\perp}} W=0
$$

where $\bar{K}(z)=K+(\lambda / 2) \int W_{00}\left(0, \mathbf{p}_{\perp}, z\right) d^{2} p_{\perp}$, and $W_{00}\left(0, \mathbf{p}_{\perp}, z\right)$ is the fundamental mode of a complete set of solutions $W_{n m}\left(\mathbf{r}_{\perp}, \mathbf{p}_{\perp}, z\right)$ of Eq. (9) evaluated in $\mathbf{r}_{\perp}=0$. It is easy to see that $W_{00}\left(0, \mathbf{p}_{\perp}, z\right)=\exp \left[-\sigma^{2}(z) p_{\perp}^{2} / \epsilon^{2}\right] /(\pi \epsilon)^{2}$, where $\sigma(z)$ satisfies the following envelope equation:

$$
\frac{d^{2} \sigma}{d z^{2}}+\bar{K}(z) \sigma-\frac{\epsilon^{2}}{\sigma^{3}}=0 .
$$

By substituting both the expression of $\bar{K}(z)$ and the one of $W_{00}\left(0, \mathbf{p}_{\perp}, z\right)$, we finally obtain the beam envelope equation that accounts for a focusing quadrupole-like interaction $(K>0)$, provided by the strong axial magnetic field, and the space charge effects in the strongly localized beam spot size, viz.,

$$
\frac{d^{2} \sigma}{d z^{2}}+K \sigma+\frac{\Gamma}{\sigma}-\frac{\epsilon^{2}}{\sigma^{3}}=0,
$$

where $\Gamma=\lambda / 2 \pi$. This is the well known Sacherer envelope equation [14] widely used in the conventional description of the beam motion in the presence of the space charge effects.

We have presented a review of the main investigations on the quantum-like description of the charged-particle beam transport provided by the TWM. The predictions of the TWM have been compared to the ones of the particle tracking simulations, showing a satisfactory agreement in absence of space charge effects. A simple analysis in terms of the Wigner quasidistribution, that have shown the possibility to correctly extend the phase space TWM description with the inclusion of the space charge effects, has been put forward.

\section{References}

[1] J.D. Lawson, 1988 The physics of charged-particle beams (Clarendon, Oxford).

[2] R. Fedele and G. Miele, Nuovo Cim. D 13, 1527 (1991).

[3] R. Fedele and P.K. Shukla, Phys. Rev. A 454045 (1992).

[4] R.Fedele and G.Miele, Phys.Rev.A 46, 6634 (1992).

[5] S. De Nicola, R. Fedele, V.I. Man'ko and G. Miele, Phys. Scr. 52, 191 (1995).

[6] R. Fedele, G. Miele, L. Palumbo and V.G. Vaccaro, Phys. Lett. A 179, 407 (1993).

[7] R. Fedele, F. Galluccio and G. Miele, Phys. Lett. A 185, 93 (1994).

[8] R. Fedele, G. Miele and L. Palumbo, Phys. Lett. A 194, 113 (1994).

[9] R. Fedele, F. Galluccio, V.I. Man'ko and G. Miele, Phys. Lett. A 209, 263 (1995).

[10] R. Fedele and M.A. Man'ko, Eur. Phys. J. D 27, 263 (2003).

[11] K. Husimi, Proc. Phys. Math. Sot. Japan 22, 264 ( 1940).

[12] Ji-ho Jang, Yong-sub Cho, Hyeok-jung Kwon, Phys. Lett. A 366, 246 (2007).

[13] Ji-ho Jang, Yong-sub Cho, Hyeok-jung Kwon, Nucl. Instr. Meth. Phys. Res. A 624, 578 (2010).

[14] F.J. Sacherer, IEEE Trans. Nucl. Sci. NS-18, 1105 (1971). 